\title{
A Suspected Atypical Case of Ramsay Hunt Syndrome Diagnosed in The Emergency Department
}

Stephen Dunay, Alexis Taylor, Tristan Knutson

Department of Emergency Medicine, Madigan Army Medical Center, Tacoma, USA

Cite this article as: Dunay S, Taylor A, Knutson T. A Suspected Atypical Case of Ramsay Hunt Syndrome Diagnosed in The Emergency Department. J Emerg Med Case Rep 2017; 8: 85-7.

\section{ABSTRACT}

Introduction: Ramsay Hunt syndrome is a rare complication of varicella zoster virus (VZV) reactivation, occurring in only approximately $0.2 \%$ of VZV reactivation cases. Despite its rarity, the morbidity associated with the syndrome is high, and the symptoms can be varied and nonspecific.

Case Report:This report presents the case a 42-year-old female with an atypical presentation of Ramsay Hunt syndrome. Initially, her symptoms were concerning with regard to stroke; however, stroke workup was normal, and the consultants considered her symptoms to be the most consistent with Ramsay Hunt syndrome.

Conclusion: The classical symptoms of Ramsay Hunt syndrome include otalgia; a vesicular rash in either the auditory canal, hard palate, or anterior two-third of the tongue; and ipsilateral facial paralysis or weakness. Our literature review revealed that there were no similar case reports; however, all emergency physicians should include this syndrome as part of the differential diagnosis of patients who present with unilateral paralysis.

Keywords: Emergency medicine, neurology, herpes zoster, Ramsay Hunt, otolaryngology

Received: 18.04.2017 Accepted: 04.05.2017 Available Online Date: 25.08.2017

\section{Introduction}

Ramsay Hunt syndrome type 2 (RHS2; also called herpes zoster oticus) is a rare complication of varicella zoster virus (VZV) reactivation, occurring in only approximately $0.2 \%$ of $V Z V$ reactivation cases (1). $V Z V$ reactivation primarily presents as a painful unilateral maculopapular vesicular rash that is limited to a dermatomal distribution with systemic symptoms such as fever or malaise, otherwise known as shingles (2). VZV usually stays dormant in the dorsal root ganglia of sensory nerves after infection with varicella (chickenpox), but it can be reactivated in the elderly and immunocompromized individuals (3). The United States Center for Disease Control reveals that approximately 32\% of Americans will have VZV reactivation during their lifetime; however, this number is expected to decrease as the number of people who have received the varicella vaccine instead of having had an active infection increases with age (1). Reactivated latent VZV in the geniculate ganglion of the facial nerve is classified as RHS2 (4).

\section{Case Report}

A 42-year-old female with chief complaints of right ear pain, nausea, and dizziness for 4 days was examined in the emergency department after she noticed a small "pimple" on the right auricle that she popped and drained at home. She also complained 
of a stiff neck and a fever blister on her lower lip. She was examined at another emergency department on the preceding day and was diagnosed with otitis externa and thus was prescribed otic ofloxacin. Despite using antibiotics, the pain worsened, and she had "muffled hearing."She did not have fever, vision changes, weakness, vertigo, or any other complaints. On that day, her vital signs were normal, and her physical examinations were notable for redness, warmth, and swelling of the right auricle, with tenderness on palpation, as well as a fever blister on the lower lip. No vesicles or pustules were noted, and her neurological examination was nonfocal. Otolaryngology service was consulted for assistance with management because of concerns of a cartilaginous spread. Examination by the otolaryngology resident was additionally notable for a Weber test that lateralized to the right ear, and air conduction greater than bone conduction bilaterally., suggesting a sensorineural hearing loss. The patient was discharged home with a prescription for ciprofloxacin because of presumed auricular cellulitis and was instructed to follow-up with her primary doctor.

After 2 days, the patient called the emergency medical services after awaking at 3:00 AM with sudden right-sided motor deficit and slurred speech. She had no neurological symptoms before going to bed. She arrived with normal vital signs, and bedside glucose levels were normal. She stated that on waking up, she was unable to close her right eye and had a right facial droop and right and left arm weakness. Bedside examination that was performed by the emergency department resident revealed a right facial droop without forehead sparing, right upper extremity drift, and mild right lower extremity drift with gravity. She reported diminished sensation in the right upper extremity and demonstrated dysarthric speech. She had sensory deficit in the cranial nerves $\mathrm{V} 1-3$ on the right and was unable to close her right eye against resistance. Her National Institute of Health $(\mathrm{NIH})$ stroke score was 8 , and thus, the stroke team was alerted.

Head computed tomography without contrast, performed while awaiting for the arrival of the neurology team, was unremarkable. The examination by the neurology resident was similar to the initial examination, and the team recommended STAT magnetic resonance imaging of the brain, which was also unremarkable. The only abnormal laboratory result was a thyroid-stimulating hormone level of 9.1; however, the patient endorsed a history of hypothyroidism and noncompliance with her prescribed levothyroxine.

Imaging effectively ruled out an acute cerebrovascular accident (CVA), and the treatment team could not determine the diagnosis. After immediate interventions to rule out stroke were performed, the neurology resident requested a more thorough history examination, and re-engaged the on-call otolaryngology residents to evaluate the patient. Despite the lack of vesicles on her examination, they believed that her symptoms were consistent with RHS. Unfortunately, her progressive facial weakness was expected to worsen, which would likely not be reversible. Additionally, her peripheral weakness could be explained as a symptom of hypothyroidism, and not RHS. She was administered 1000 mg valacyclovir three times daily for 7 days, a steroid taper, and lacrilube with eye taping at night.

\section{Discussion}

The classical symptoms of RHS2 include otalgia; vesicular rash in either the auditory canal, hard palate, or anterior two-thirds of the tongue; and ipsilateral facial paralysis or weakness (1). Although this is a clinical diagnosis, testing for VZV antibodies in the patient's blood may be helpful, particularly whether the patient had varicella in the past is unknown (5). RHS2 may be confused with Bell's palsy, especially because the rash is not always present when paralysis starts. VZV reactivation may rarely present with facial paralysis and no vesicular rash, known as zoster sine herpete (6). However, paralysis in RHS2 tends to be more severe and is less likely to resolve. Treatment includes antivirals and possibly steroids. Without treatment, only $20 \%$ of patients will have complete resolution of facial paralysis (7).

Although RHS2 is rare, there are several variations from its classical presentation. An extremely rare presentation of RHS2 includes cerebellar involvement. These patients experience limb dysmetria, gait ataxia, and horizontal nystagmus, along with otalgia and right vesicular ear rash. Of note, the patient did not have a history of either varicella infection or vaccination but was diagnosed after VZV was detected in her cerebrospinal fluid by polymerase chain reaction (8). In addition, RHS2 can present with concomitant cervical VZV reactivation in addition to facial nerve symptoms $(1,9)$. Bharadwaj et al. (3) described a case of RHS2 that was atypical for two reasons: the patient presented with cranial nerve polyneuritis (usually only one cranial nerve is affected) and bulbar symptoms (dysphagia and hoarseness) that mimicked bulbar stroke.

\section{Conclusion}

Our case was unique because the patient presented with symptoms for CVA with an NIH stroke score of 8, which prompted calling a code stroke.

She exhibited right-sided weakness, right upper extremity greater than right lower extremity drift with gravity, decreased right upper extremity sensation, increased right lower leg sensation, and dysarthria. In addition, her extremity symptoms appeared to resolve within several hours after waking up with these concerning symptoms. There have been case reports of ophthalmic herpes zoster presenting with delayed contralateral hemiparesis that mimics stroke; however, there has been no report of this occurring with RHS2 (6). This unusual presentation of RHS2 highlights the importance of including RHS2 in the differential diagnosis of a patient with CVA symptoms if they experience ear pain or have signs of a vesicular rash on examination.

Informed Consent: Written informed consent was obtained from the patient who participated in this study.

Peer-review: Externally peer-reviewed.

Author Contributions: Supervision - T.K.; Literature Search - S.D., A.T.; Writing Manuscript - S.D., A.T., T.K.; Critical Review - T.K.

Conflict of Interest: No conflict of interest was declared by the authors. 
Financial Disclosure: The authors declared that this study has received no financial support.

\section{References}

1. Worme M, Reena Chada R, Lavallee L. An unexpected case of Ramsay hunt syndrome: case report and literature review. BMC Res Notes 2013; 6: 337. [CrossRef]

2. Ferri FF. Herpes Zoster. Ferri's Clinical Advisor 2017; 587-96.

3. Bharadwaj S, Moffat AC, Wood B, Bharadwaj A. Herpetic cranial polyneuritis mimicking brain stem infarction-an atypical presentation of Ramsay Hunt syndrome. BMJ Case Rep 2016. [CrossRef]

4. Sweeney CJ, Gilden DH. Ramsay Hunt syndrome. J Neurol Neurosurg Psychiatry 2001; 71: 149-54. [CrossRef]
5. Gupta NM, Parikh MP, Panginikkod S, Gopalakrishnan V. Ramsay Hunt Syndrome. QJM 2016; 109: 693. [CrossRef]

6. Volpi A. Severe Complications of Herpes Zoster. Herpes 2007; 14: 35-9.

7. Murakami S, Hato N, Horiuchi J Honda N, Gyo K, Yanagihara N. Treatment of Ramsay Hunt Syndrome with Acyclovir-Prednisone: Aignificance of Early Diagnosis and Treatment. Ann Neurol 1997; 41: 353-7. [CrossRef]

8. Chan TL, Cartagena AM, Bombassaro AM, Hosseini-Moghaddam SM. Ramsay Hunt Syndrome Associated with Central Nervous System Involvement in an Adult. Can J Infect Dis Med Microbiol 2016. [CrossRef]

9. Kayayurt K, Yavasi O, Bilir O. Ersunan G, Giakoup B. A Case of Ramsay Hunt Syndrome with Atypical Presentation. Turk J Emerg Med 2016; 14: 142-5. [CrossRef] 